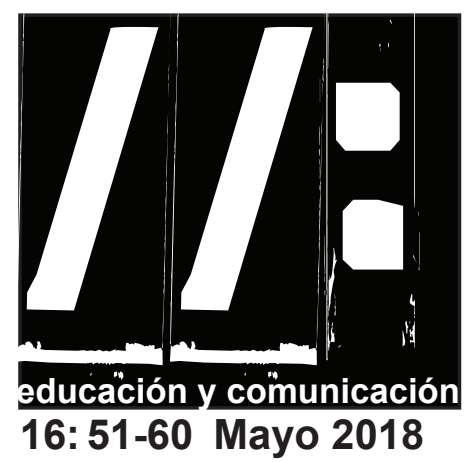

16: 51-60 Mayo 2018

\section{ANÁLISIS DEL EFECTO DE LA IMPLEMENTACIÓN DE LAS TECHOLOGÍAS DE INFORMACIÓN Y COMUNICACIÓN [TIC] EN EDUCACIÓN INFANTIL}

\section{Analysis of the effect of the implementation of Information and Communication Technologies [ICT] in children's education}

\author{
Alba Martín Ruiz-Berdejo \\ Psicologa y doctorada en el Programa de Ciencias de la Salud \\ 'Psicología, Educación y Salud', de la Universidad de Cádiz \\ (España) \\ E.mail: albamartinpsicologa@gmail.com
}

\title{
Resumen:
}

Actualmente las tecnologías de la información y comunicación (TIC) están implementadas en la mayoría de las aulas españolas de Educación Infantil. El objetivo del presente artículo es determinar en qué medida afectan las TIC al aprendizaje infantil. Nos centramos en el uso de la Tableta (Tablets) y Realidad Aumentada (RA) al ser las más frecuentadas en las aulas infantiles. Por otro lado, damos a conocer los proyectos digitales españoles y mundiales más recientes. Por último, vemos el papel del docente respecto a las TIC y realizamos una reflexión profunda sobre la relación nuevas tecnologías y enseñanza.

Palabras clave: TIC, educación infantil, nativos digitales, Tableta, realidad aumentada.

\section{Abstract:}

Currently, information and communication technologies (ICT) are implemented in most of the Spanish classrooms for early childhood education. The objective of this article is to determine how ICTs affect children's learning. We focus on the use of the Tablet and Augmented Reality (AR) as they are the most frequented in children's classrooms. On the other hand, we present the most recent Spanish and world digital projects. Finally, we see the role of the teacher with regard to ICT and we carry out a deep reflection on the relationship between new technologies and teaching. Keywords: ICT, early childhood education, digital natives, tablet, augmented reality. 


\section{II: Educación infantil: siglos XX y XXI}

\section{Introducción}

6 Los hijos traen un pan bajo el brazo' es un dicho popular español que podríamos sustituir perfectamente por 'los hijos traen una tableta bajo el brazo'. Sí, las nuevas tecnologías están ya implantadas en nuestra rutina, y hacemos uso de ellas como si siempre hubieran formado parte de nuestras vidas. A veces, incluso, le damos el valor como si se tratase de una necesidad primaria. Como cabría esperar, su presencia también se hace notable en las aulas de nuestros colegios, desde edad infantil hasta la Universidad. Hoy el aprendizaje con instrumentos digitales portátiles, trasciende en todas las actividades cotidianas vinculadas con el conocimiento (Castro y otros, 2016). ¿En qué medida se benefician los estudiantes de las herramientas tecnológicas? ¿Qué impacto ha tenido en el modo de procesar la información, así como en la transmisión de conocimientos y en definitiva, de la enseñanza? ¿Se hace un buen uso en el aula de las nuevas tecnologías? A lo largo del presente artículo se pretende dar respuestas a dichas preguntas, girando en torno al núcleo principal que nos atañe: la educación infantil. Así, empezaremos contextualizándonos en el concepto de nativo digital. Posteriormente definiremos que son las TIC y sus tipos, ya que cada año surgen nuevas aplicaciones, así como veremos su implementación en España. Analizaremos los proyectos más novedosos basados en las TIC en aulas, y el papel de docentes y alumnado con respecto a ello. Por último, en las conclusiones finales se expondrán reflexiones basadas en trabajos de evidencia científica, junto con líneas de investigaciones futuras.

\section{Nativos vs inmigrantes digitales.}

Es importante, antes de adentrarnos en el mundo de las TIC, entender el momento social y tecnológico en el que ha nacido nuestro alumnado. Así, surge el término de 'nativo digital' (Prensky, 2001): hablantes nativos de un lenguaje digital, mientras que al resto los define como inmigrantes digitales, personas que aunque pueden llegar a adaptarse y aprender a utilizar estas tecnologías, no dejan de ser inmigrantes en un mundo digital. Prensky considera que estos individuos no piensan ni procesan la información de la misma manera que sus predecesores en las generaciones anteriores, ya que sus patrones de pensamiento han cambiado. Otros autores, como Pedró (2006) denomina a los nativos digitales como "Aprendices del Nuevo Milenio" (en inglés, New Millennium Learners- NML).

De lo explicado anteriormente, podemos extraer la idea de que los nativos digitales aprenden más fácilmente a través de interfaces digitales, ya que las pantallas son más amigables para ellos (Pedró, 2006). A continuación, se enumeran aquellos principios neurocognitivos que brindan apoyo y permiten utilizar en forma correcta las interfaces digitales (Falco, 2017):

- 1. Principio de Relevancia: la efectividad en una comunicación se incrementa cuando no se presenta ni mucha ni poca información, debido a que un individuo entiende y recuerda más fácilmente cuando la cantidad de detalles que se utilizan para explicar un mensaje es justa y adecuada.

- 2. Principio del Conocimiento Apropiado: un individuo comprende y recuerda más fácilmente un mensaje si éste está conectado con los conoci- 
mientos previos de los conceptos, jerga y símbolos pertinentes.

- 3. Principio de Notabilidad: la atención es captada y dirigida hacia grandes diferencias perceptibles.

- 4. Principio de Discriminabilidad: para que dos propiedades puedan ser distinguidas, deben diferir por una gran proporción, es decir que un individuo requiere de contraste para diferenciar formas, colores o posiciones entre distintos objetos o con el fondo.

- 5. Principio de Organización Perceptual: los seres humanos agrupan automáticamente elementos en unidades a las cuales prestan atención y recuerdan más fácilmente que si estuvieran en forma aislada.

- 6. Principio de Compatibilidad: un mensaje es mucho más fácil de entender si es compatible con su significado. En otras palabras, aquí se aplica el refrán de que se tiende a juzgar un libro por su tapa, y en este caso, es el cerebro el que lo lleva a cabo.

- 7. Principio de Cambios Informativos: las personas esperan que los cambios en las propiedades transporten información, es decir: esperan que cada pieza de información esté indicada por un cambio en una propiedad perceptible.

- 8. Principio de la Limitación de Capacidades: el ser humano presenta una capacidad limitada para procesar información, lo cual hace que directamente no logrará entender un mensaje si demasiada información tiene que ser procesada o recordada

\section{TIC: Tabletas y Realidad Aumentada (RA)}

Las TIC tienen sus orígenes en las llamadas Tecnologías de la Información (Information Technologies o IT), concepto aparecido en la década de los setenta, el cual se refiere a las tecnologías para el procesamiento de la información (Barrías, 2017). Los medios más actuales en los que se basan las TIC son las tabletas, sirviéndose de la Realidad Aumentada (Johnson y otros, 2016).

La tableta se puede entender como un tipo de ordenador/computadora portátil integrado en una pantalla táctil de fácil manejo para el alumnado de Educación Infantil que permite un aprendizaje más eficaz (Jiménez y otros, 2017). También se utiliza, como juguete preferido donde el alumnado de temprana edad lo maneja cada vez más con gran soltura (Raposo, 2016). A pesar de que hayan algunos estudios, en concreto el titulado "Digital Game-based Learning for Early Childhood" (Peirce, 2013), realizado en Irlanda, que concluye en que los beneficios del uso de las tabletas a través de juegos educativos informatizados, son más bien escasos o anecdóticos; sin embargo, y a falta de resultados de nuevos estudios que lo puedan constatar, sí que se valoran sus posibilidades en áreas de la conciencia fonológica, las relaciones diferenciales, el estímulo de la memoria, las habilidades motoras coordinadas y en el desarrollo de destrezas matemáticas. En esta línea, se sitúa el contenido del informe sobre las tabletas en Educación Infantil (Marqués, 2014), donde se resalta el impacto positivo de su uso en la escuela para que los niños y niñas mejoren su comprensión, creatividad, capacidad de memoria, motivación para aprender, desarrollo de competencias digitales y el aprendizaje autónomo. 
El ambiente de rigidez de la enseñanza tradicional contrasta con el aprendizaje basado en juegos y la narración de historias, «storytelling», como vías especialmente prominentes para generar motivación extrínseca (Cadavieco y otros, 2017). Otros autores comparan el aprendizaje móvil con el tradicional y, aunque no encuentran diferencias significativas, consideran que su sentido de juego resulta más agradable, ya que une los desafíos lúdicos con la rigidez del mundo real, conceptos abstractos con los retos prácticos, procesos de aprendizaje en contextos verídicos y contenidos virtuales en RA (Furió y otros, 2015). Otras investigaciones verifican el potencial atractivo de la RA para los estudiantes (Cubillo otros, 2015). La RA ha demostrado tener la capacidad de proporcionar al alumnado una mejor comprensión de conceptos como el espacio tridimensional, formas geométricas, determinadas áreas matemático-geométricas (De la Torre y otros, 2013; Cubillo y otros, 2014). Otras investigaciones exploran la involucración emocional de alumnos/as que aprendieron acerca de la Guerra Mundial con el acercamiento multimodal de la RA (Sakr y otros, 2016). Mientras que otros estudios combinan estas ideas en una plataforma de RA, donde generan juegos de aprendizaje de ciencias que interaccionan con el contexto (Laine y otros, 2016). Por último, parece que el aprendizaje fuera del centro educativo no suele lograr un profundo cambio en los comportamientos y patrones generados, y es difícil controlar su eficacia (Cadavieco y otros, 2017). En este sentido, la RA destaca la sustitución de la experiencia del trabajo de campo al realizarse en el propio dispositivo (Harley y otros, 2016), entrando en ambientes inmersivos e interactivos, aulas virtuales o escenarios diseñados para apoyar el aprendizaje (Nagatay otros,
2016). Otros autores proponen un algoritmo científico para identificar objetos de la realidad a los cuales puede agregarse la RA para usos educativos (Tan y otros, 2015). También, otros grupos de investigación desarrollan una nueva metodología que reproduce un sistema ecológico virtual semejante a un jardín donde los estudiantes interactúan y ven crecer insectos (Tarng y otros, 2015).

\section{Sistema Educativo Español: Proyectos innovadores.}

En la última actualización de la reforma educativa que se establece con la Ley Orgánica de Mejora de la Calidad Educativa (LOMCE), publicada como Ley Orgánica 8/2013, de 9 de diciembre, y que entró en vigor el día 30 de diciembre de 2013, en su preámbulo, se comenta que los cambios en la sociedad y la globalización provocan que "necesitamos propiciar las condiciones que permitan el oportuno cambio metodológico, de forma que el alumnado sea un elemento activo en el proceso de aprendizaje". Además, se indica el gran cambio que se está produciendo en los métodos de enseñanza: "La globalización y el impacto de las nuevas tecnologías hacen que sea distinta su manera de aprender, de comunicarse, de concentrar su atención o de abordar una tarea". Así, todos los centros educativos han de tener como misión dicha incorporación de las TIC.

En la literatura revisada en el presente año, destaca como practicas basada en las TIC el Proyecto ACRA (Martínez y otros, 2018) dirigido por el equipo de investigación TECN@ de la Universidad de Oviedo. Es un proyecto innovador centrado en la utilización didáctica de la RA para potenciar el desarrollo y la 
adquisición de contenidos científicos en el alumnado de Educación Infantil, Primaria, Secundaria y Bachillerato, en el cual participaron 9 centros educativos ubicados en diferentes zonas geográficas de Asturias, y se planificaron y desarrollaron veintiuna experiencias (Martínez y otros, 2018).

Por otro lado, destaca el proyecto 'Nuevas voces ¡Nuevos Retos!' del Colegio Purísima para niños sordos (Zaragoza), ganador del Premio SIMO EDUCACIÓN Categoría Educación infantil de 2017. Para ayudar a su alumnado a escuchar y hablar mejor, en este colegio llevan a cabo la rehabilitación auditiva y lingüística mediante la robótica y la programación, utilizando el Método Verbotonal con apps en iPad y algunos robots.

En la Web de la revista 'Educación 3.0' (2017) encontramos un artículo donde se recogen 35 prácticas basadas en las TIC en Educación Infantil en aulas españolas en los últimos años.

\section{El papel de los docentes con respecto a las TIC.}

Hasta ahora todo son ventajas de las TIC pero... ¿qué pasa con la labor docente? Se vive un clima de innovación, de cambio, pero por el momento no se concreta más que en modificaciones ocasionales de las actividades y, sobre todo, en una actitud del alumnado claramente más favorable al desarrollo de las tareas académicas en las que se utiliza la herramienta del ordenador (Pérez y Sola, 2006). Parece que introducir la tecnología en las escuelas por sí sola, sin ningún proyecto pedagógico donde los papeles están bien definidos, no produce estudiantes más reflexivos y críticos, ni mejora los aprendizajes (Area, 2005), de ahí la importancia de la figura del profesor, el cual es el responsable de hacer un uso útil y significativo de las tabletas y demás aparatos electrónicos para mejorar el aprendizaje del alumnado.

La implementación de las TIC es un reto para el cuerpo docente (Burden y otros, 2016), así varias investigaciones alertan sobre la necesidad de explorar continuamente las bondades o intereses que las impulsan. De hecho, aunque se tiene una percepción positiva y buena imagen de las TIC en el aula, por parte de los cuestionarios reportados del profesorado (Barría ,2017), es cierto que la bibliografía nos muestra que a nivel pedagógico, no se ha avanzado tanto.

Para Gewerc y otros (2015), la competencia digital implica el desarrollo del razonamiento crítico en el profesorado, el dominio de diferentes lenguajes específicos (audiovisual y textual) así como el uso de las TIC como vehículos de información, comunicación y producción de conocimiento; supone uno de los factores que influyen en la toma de decisiones del profesorado sobre el uso educativo de las TIC (Badia y otros, 2013). Por ello, y desde el proceso de formación inicial docente, se debe incorporar dicha competencia de manera trasversal, desde distintas materias, para así favorecer su desempeño como "competente digital" en el uso didáctico de las TIC y su explotación en el aula inclusiva (Lozano y otros, 2014). Además, la formación no sólo debe centrarse en la presentación y exploración de las herramientas y software, sino también en la promoción de actitudes y eficacia docente para realizar la transferencia de información al alumnado (Gegenfurtner, 2011). El profesorado debe conocer nuevos métodos de aprendizaje basados en este tipo de recursos y el procedimiento para seleccionar herramientas apropiadas con las que conseguir las metas de aprendizaje (Ertmer y otros, 2010). Otros 
autores (Espuny y otros, 2012) identifican como factor de éxito para la integración de las TIC en el aula la existencia de docentes investigadores o emprendedores, capaces de fomentar el uso frecuente de tecnologías digitales en el aula, pues permite el trabajo en red, la coordinación y canalización de las decisiones docentes (Pérez y otros, 2011).

Cabe decir que desde el Ministerio de Educación, Cultura y Deporte Español se ofrece recursos para los docentes en el ámbito de las TIC a través del proyecto 'Educa Lab'. Tal y como se describe en la web, 'Educalab' es un lugar de encuentro para la educación. $\mathrm{Su}$ objetivo es apoyar al profesorado y en sentido amplio a todo el sistema educativo español desde el conocimiento y la cercanía, desde los datos y el análisis y desde la investigación, la experimentación y la innovación. Otras plataformas de interés son: eTwinning, Aula del Futuro o Scientix .

\section{Consideraciones finales}

A lo largo del presente artículo hemos analizado el paronama actual de las TIC en las aulas de Educación Infantil españolas. A pesar de que las tecnologías están implantadas a todos los niveles educativos, vemos como hay un menor número de proyectos en la literatura (Barría, 2017). Puede que uno de los motivos sea el propio estadio evolutivo y cognitivo de los niños y las niñas en estas edades, o que aún no se haya adaptado la pedagogía a la tecnología. Aunque en el último año (2017) de la convocatoria SIMO (Salón de Tecnología para la Enseñanza) se recibió un 45\% más de solicitudes que el año 2015 para exponer los proyectos realizados con TIC, solo seis experiencias se desarrollaron en Educación Infantil, siendo un mayor número en Educación Primaria.
$Y$ es que mientras la tecnología avanza a pasos de gigante, ya que cada mes se lanza al mercado nuevos aparatos electrónicos, la educación vive ahora un cambio de paradigma de enseñanza el cual lleva años fraguándose. De hecho, es una de las principales ideas que se expone en el apartado de docentes: se necesita un protocolo e innovar pedagógicamente con respecto a la TIC. Será imprescindible para el profesorado comprender e instruirse en el aspecto cognitivo del aprendizaje (Falco y otros, 2016), no solo para especificar la función de las tecnologías a partir de la teoría cognitiva, sino como un medio para entender fehacientemente cómo funciona el cerebro cuando el alumnado aprende por vía de las TIC. En este sentido, señala Gros (2004), que "no siempre el uso de la tecnología conduce a la innovación y la reflexión sobre el aprendizaje" y debemos partir de que "el objetivo no es usar la tecnología, sino adaptar la educación a las necesidades actuales, $y$, por tanto, se precisa un cambio metodológico" Gros (2004). De ahí se desprende la idea de que quizás hemos cambiado la forma de educar y transmitir conocimientos, pero el contenido sigue siendo el mismo.

Es decir, cambia el canal, pero no el mensaje, ni el código: se siguen proyectando producciones animadas de Disney, pero en lugar de hacerlo a través de una cinta VHS, se almacena en la nube y se proyecta en tabletas o pantalla digital. En definitiva, innovación tecnológica debe de ir de la mano de innovación pedagógica.

A pesar de las ventajas innumerables que hasta ahora hemos citado de las TIC, debemos prestar atención al uso que hace nuestro alumnado. Aunque el empleo de tecnologías aumenta, la disposición de los estudiantes a aprender más sobre el entorno, también se ha considerado el carácter problemático que conlleva el 
uso del dispositivo móvil en el aula. Las TIC puede romper la normalidad del aula, y habitualmente sucede en los exámenes (Kaiiali y otros, 2016). Otros autores han incidido en cómo el uso de la telefonía móvil en la escuela es problemático y muchos docentes son reticentes a su uso al desviar la atención, generar ciberacoso y otras problemáticas. Además, parece que la penetración de las TIC va relacionada con las infraestructuras. Burden y Hopkins (2016) identifican como barreras para la inserción de las tecnologías el contexto físico y la capacitación del personal, como barreras secundarias las actitudes y creencias.

Hay una visión esperanzadora de avance pedagógico, en el sentido de que es una línea de investigación fértil y fructífera. Así, aunque el proceso es lento, aparecen nuevos conceptos pedagógicos como el flipped classroom ("clase invertida"), donde el alumnado hace «deberes» en clase junto con el profesorado y el resto de compañeros/as, y aprende la teoría y las lecciones en casa (Lax y otros, 2017) o la gamificación, donde se potencian juegos interactivos para aprender jugando y sin apenas darse cuenta (Díaz, 2017). En relación con este último concepto, se está empezando a usar recientemente la RA como medio facilitador de la gamificación.

La incorporación de las competencias básicas al currículo ha significado muchos cambios en la educación europea, en el caso de España específicamente, ha tenido que pasar de un diseño curricular de carácter académico, basado en la consecución de objetivos de conocimiento, a otro de carácter más práctico (Barría, 2017). Por este cambio de enfoque, algunos autores (Castillo y otros, 2010) proponen denominar proceso de aprendizaje-enseñanza al proceso de enseñanza-aprendizaje. La diferencia se encuentra en que anteriormente el profesor era el protagonista, el que entregaba el conocimiento en lecciones magistrales; mientras que el nuevo enfoque convierte al alumnado en protagonista de su propio aprendizaje y el profesorado cumple con la labor de guía, asesoría, orientación y facilitación de recursos y herramientas. Es por eso la diferenciación que hoy se plantea para dicho proceso, anteponiendo el aprendizaje del alumnado sobre la enseñanza magistral (Castillo y otros, 2010). Todo ello, con la ilusión y la esperanza puesta en la inminente llegada de una generación de profesores y profesoras, de maestros y maestras que son nativos digitales, al igual que su alumnado.

\section{Referencias}

Area, M. (2005). Las tecnologías de la información y comunicación en el sistema escolar: una revisión de las líneas de investigación. Revista Electrónica de Investigación y Evaluación Educativa, 11.

Badia, A.; Meneses, J. y otros (2013). Percepción de los docentes sobre los factores que afectan el uso educativo de las TIC en el aula equipada de tecnología. Electronic Journal of Research in Educational Psychology, 11.

Barría, C. (2017). La incorporación de las TIC en los centros educativos y sus efectos educativos en los procesos de aprendizaje y enseñanza. Granada: Universidad de Granada.

Burden, K. \& Hopkins, P. (2016). Barriers and Challenges Facing Pre-Service Teachers use of Mobile Technologies for Teaching and Learning. International Journal of Mobile and Blended Learning, 8; 2 1-20.

Cadavieco, J. F.; Sevillano, M. A. P. y otros (2017). 


\section{II: Educación infantil: siglos XX y XXI}

M-learning y realidad aumentada: Revisión de literatura científica en el repositorio WoS. Comunicar: Revista científica iberoamericana de comunicación y educación, 52; 63-72.

Castillo, S. y Cabrerizo, J. (2010). Evaluación educativa de aprendizajes y competencias. Madrid: Pearson Educación.

Castro, G.; Dominguez, E. y otros (2016). Mobilearn: Context-aware mobile learning system. IEEE Latin America Transactions, 14; 958-964.

Cubillo, J.; Martin, S. \& otros (2015). Preparing augmented reality learning content should be easy: UNED ARLE - An authoring tool for augmented reality learning environments. Computer Applications in Engineering Education, 23; 778-789.

Cubillo, J.; Martín, S. y otros (2014). A learning environment for augmented reality mobile earning. En Frontiers in Education Conference (FIE). República Checa: European Schoolnet.

De la Torre, J.; Martin-Dorta, N. y otros (2015). Entorno de aprendizaje ubicuo con realidad aumentada y tabletas para estimular la comprensión del espacio tridimensional. Revista de Educación a Distancia, 37. Díaz, P. P. (2017). Gamificando con Kahoot en evaluación formativa. Revista Infancia, Educación y Aprendizaje, 3; 112-117.

Educación 3.0 (2017). Infantil: 35 buenas prácticas educativas con TIC. Recuperado de: https://www. educaciontrespuntocero.com/experiencias/infantilbuenas-practicas-educativas-con-tic/26080.html.

Ertmer, P. A. y Ottenbreit-Leftwich, A. T. (2010). Teacher technology change: How knowledge, confidence, beliefs, and culture intersect. Journal of researchon Technology in Education, 42; 255-284.

Espuny, C.; Gisbert , M. y otros (2012). El coordina- dor TIC en los centros educativos: funciones para la dinamización e incorporación didáctica de las TIC en las actividades de aprendizaje. Pixel-bit. Revista de Medios y Educación, 41

Falco, M. (2017). Reconsiderando las prácticas educativas: TICs en el proceso de enseñanza-aprendizaje. Tendencias pedagógicas, 29; 59-76

Furió, D. Juan, M. C. y Vivó, R. (2015). Mobile learning vs. traditional classroom lessons: a comparative study. Journal of Computer Assisted Learning, 31; 189-201.

Gegenfurtner, A. (2011). Motivation and transfer in professional training: A meta-analysis of the moderating effects of knowledge type, instruction, and assessment conditions. Educational Research Review, 6; 153-168.

Gewerc, A. y Montero, L. (2015). Conocimiento profesional y competencia digital en la formación del profesorado. El caso del Grado de Maestro en Educación Primaria. Revista Latinoamericana de Tecnología Educativa, 14; 31-43.

Gros, B. (2004). La construcción del conocimiento en la red: límites y posibilidades. Revista electrónica Universidad de Salamanca, 5.

Harley, J.; Poitras, E. y otros (2016). Comparing Virtual and Location-Based Augmented Reality Mobile Learning: Emotions and Learning Outcomes. Educational Technology Research and Development, 64: 359-388.

Jiménez, E. R. Galán, R. P. y Torres, N. Q. (2017). Utilización de tablets en Educación Infantil: Un estudio de caso/Use of the tablets in Early Childhood Education: A case study. Revista Latinoamericana de Tecnología Educativa-RELATEC, 16; 193-203.

Johnson, L.; Adams, S. y otros (2016). NMC Horizon 
Report: 2016. Higher Education Edition. Austin: The New Media Consortium.

Kaiiali, M.; Ozkaya, A. y otros (2016). Designing a Secure Exam Management System (SEMS) for MLearning Environments. IEEE Transactionson Learning Technologies, 9; 258-271.

Kuz, A.; Falco, M. y Giandini, R. (2016). Análisis de redes sociales: un caso práctico. Computación y Sistemas, 20; 89-106.

Laine, T. Nygren, E. y otros (2016). Science Spots AR: A Platform for Science Learning Games with Augmented Reality. ETR\&D - Educational Technology Research and Development, 64; 507-531.

Lax, N. Morris, J. y Kolber, B. J. (2017). A partial flipclassroom exercise in a large introductory general biology course increases performance at multiple levels. Journal of Biological Education, 51; 412-426.

Lozano, J. Castillo, I. S. y Veas, A. (2014). Alumnado con necesidades específicas de apoyo educativo de Educación Secundaria Obligatoria y consumo de las TIC en la Región de Murcia. Etic@ net, 14; 57-69.

Marquès, P. (2014). Metainvestigación 2013-14. Uso educativo de las tabletas digitales en Educación Infantil. Informe final. Recuperado de: https:/www. slideshare.net/peremarques/uso-educativo-de-las-tabletasdigitalesen-educacin-infantil

Martínez, L. V.; Pérez, M. y otros (2018). Proyecto ACRA: experiencias didácticas en ciencias con realidad aumentada en los niveles pre-universitarios. Edutec. Revista Electrónica de Tecnología Educativa. 62; 1-18.

Ministerio de Educación, Cultura y Deporte de España (2013). Ley Orgánica 8/2013, de 9 de diciembre, para la mejora de la calidad educativa. Boletín Oficial del
Estado. Recuperado de: www. boe. es/diario_boe/txt. Php. Ministerio de Educación, Cultura y Deporte de España. Proyecto Educa Lab. Recuperado de: http://educalab.es/home

Nagata, J. Giner, J. y Abad, F. (2016). Virtual Heritage of the Territory: Design and Implementation of Educational Resources in Augmented Reality and Mobile Pedestrian Navigation. IEEE-RITA, 11; 41-46.

Pedró, F. (2006). Aprender en el nuevo milenio: Un desafío a nuestra visión de las tecnologías y la enseñanza. Recuperado de: http://educarchile.cl/UserFiles/P0001/File/nmlesp.pdf

Peirce, N. (2013). Digital game-based learning for early childhood. A state of the art report. Dublin: Learnovate Centre.

Pérez, A. I. y Sola, M. (2006). La emergencia de buenas prácticas. Informe final. Sevilla: Junta de Andalucía, Consejería de Educación. Dirección General de Innovación Educativa y Formación del profesorado. Pérez, M.; Hernando-Gómez, Á. y otros (2011). La integración de las TIC en los centros educativos: percepciones de los coordinadores y directores. Estudios pedagógicos, 37; 197-211.

Prensky, M. (2001). Digital natives, digital immigrants part 1. On the horizon, 9; 1-6.

Raposo, M. (2016). Experiencias de innovación con TIC. En Gallego, M.J. y Raposo, M.(Coords.). Formación para la educación con tecnologías. Madrid: Pirámide; 200-250.

Sakr, M. Jewitt, C. y Price, S. (2016). Mobile experiences of historical place: A multimodal analysis of emotional engagement. Journal of the Learning Sciences, 25; 51-92.

Tan, Q. y Chang, W. (2015). Location-Based Aug- 


\section{II: Educación infantil: siglos XX y XXI}

mented Reality for Mobile Learning: Algorithm, System and Implementation. Electronic Journal of eLearning, 13; 138-148.

Tarng, W.; Ou, K, y otros (2015). Development of a Virtual Butterfly Ecological System Basedon Augmented Reality and Mobile Learning Technologies. Virtual Reality, 19; 253-266. 